\title{
V-ANFIS for Dealing with Visual Uncertainty for Force Estimation in Robotic Surgery
}

\author{
Angelica I. Aviles ${ }^{1}$ Samar M. Alsaleh ${ }^{2}$ Eduard Montseny $^{3}$ and Alicia Casals ${ }^{1,4}$ \\ ${ }^{1}$ Intelligent Robotics and Systems Group, Universitat Politècnica de Cataluya, Spain \\ ${ }^{2}$ Bioinformatics and Biomedical Computing Group, George Washington University, U.S.A. \\ ${ }^{3}$ Automatic Control Department, Universitat Politècnica de Cataluya, Spain \\ ${ }^{4}$ Institute for Bioengineering of Catalonia, Spain
}

\begin{abstract}
Accurate and robust estimation of applied forces in Robotic-Assisted Minimally Invasive Surgery is a very challenging task. Many vision-based solutions attempt to estimate the force by measuring the surface deformation after contacting the surgical tool. However, visual uncertainty, due to tool occlusion, is a major concern and can highly affect the results' precision. In this paper, a novel design of an adaptive neuro-fuzzy inference strategy with a voting step (V-ANFIS) is used to accommodate with this loss of information. Experimental results show a significant accuracy improvement from 50\% to $77 \%$ with respect to other proposals.
\end{abstract}

Keywords: soft computing, robotic-assisted surgery, force estimation

\section{Introduction}

Robotic-Assisted minimally invasive surgery (RAMIS) systems emerged from the need to address some deficiencies associated with traditional Minimally Invasive Surgery (MIS) [1]. However, existing RAMIS Systems do not provide haptic feedback, which means that surgeons lose the sense of touch and that decreases their efficiency and can result in collateral tissue damage [2]-[5]. Several studies have shown that haptic feedback is able to increase the precision of telesurgery by reducing trauma and interaction forces with the tissue. It can also reduce the time needed to complete the task while maintaining high accuracy with less error [6]-[8].

Research for restoring haptic feedback in RAMIS has mainly been focused on the development of instrument-tissue force sensors that are located at the distal end either close to, or on, the instrument tip [9]-[11]. However biocompatibility and sterilization constraints, size and high cost of the devices, and the difficulty of adapting them to the tool puts severe restrictions on their use in real clinical environments [12], [13]. Therefore, new methods, capable of avoiding the use of force sensors, have recently become very prevalent.

Currently, estimation of the applied forces is the most promising approach suitable for clinical adoption. Some of the studies tackled the issue by utilizing visual information during the estimation process. In such approaches, visual displacement of contact point is mapped into an estimated force value taking into account the physical properties of the deformable object. For example, researchers in [14] and [15] used image processing to map visual clues of contour displacement into a haptic feedback estimate. In 3D simulated environments, researchers in [16] used a combination of the extended Kalman filter (EKF) and a Lyapunov-based adaptation law to estimate applied forces. The use of 3D nonlinear mass-spring-damper, along with snakes based deformable visual tracking, was investigated in [17]. Additionally, monocular endoscope images were used in [18] to construct a virtual template in which surface deformation can be modeled and mapped into a force measure.

Although the existing vision-based approaches offer robust and remote force estimation solutions, they suffer from computational complexity and limited accuracy [19]. In the search for more accurate, robust and tractable solutions that allow dealing with visual uncertainty, some researchers have made use of soft computing techniques to measure the tool-tissue force in real-time. Karimirad et al. [20] introduced a Neural Network (NN) model that, in conjunction with a motion tracking algorithm, is capable of accurately estimating the applied force to a $2 \mathrm{D}$ biological cell. In a previous work [21], we presented a Recurrent Neural Network (RNN) approach for $3 \mathrm{D}$ force estimation in RAMIS scenarios. Results showed improved accuracy compared to state of the art approaches in 3D settings, and proved the feasibility of using soft computing techniques. Apart from NNs, Fuzzy logic theory is another soft computing branch that has proven to be very useful for designing disease specific and decision theoretic expert systems for common diseases [22], [23].

Fuzzy logic and neural networks are complementary paradigms and when brought together can provide efficient intelligent systems. Neuro-Fuzzy (NF) models incorporate the generic advantages of artificial neural networks in modeling imprecise data and qualitative knowledge as well 


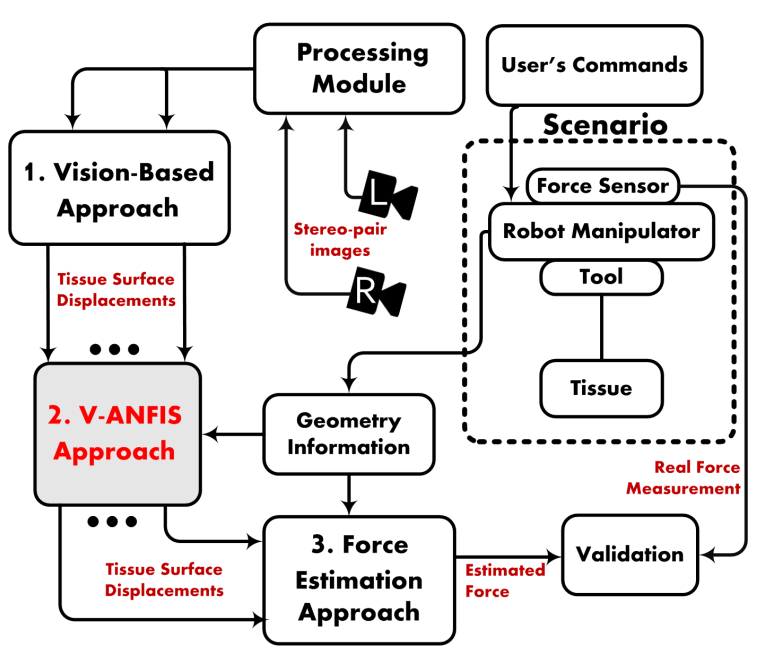

Figure 1: Proposed scheme for the estimation of the applied forces in RAMIS scenarios.

as transmission of uncertainty. One of the most powerful types of NF systems is the Adaptive Neuro-Fuzzy Inference System (ANFIS) [24], which has shown very good learning, classification, diagnosis and prediction capabilities in different medical diseases/applications [25]-[29]. In this work a Voting-Adaptive Neuro-Fuzzy Inference System (V-ANFIS) approach is proposed to improve force estimation in a RAMIS scenario. The use of ANFIS will allow handling visual uncertainty and occlusion problems to derive fast, robust and accurate predictive model for estimating the tool-tissue applied force. Moreover, the Voting process will reduce the error that occurs at the surrounding of the contact point and thereby improve the final results. To our knowledge, the work by Mozaffari et al. [30] is the only one that uses ANFIS to model tool-tissue force in RAMIS. However, that work presents three main drawbacks compared to our proposed approach: it simulates the tissue-surgical tool interaction using the computationally complex finite element method; it does not conduct $3 \mathrm{D}$ reconstruction, rather it adds the third dimension of the tool direction to the 2D simulation; and the test was not conducted in real environment as the authors only provide simulation results.

\section{Deformation Structure}

The setup for estimating the applied forces is part of the robotic system given in Figure 1 in which three main modules can be identified. The first module is a vision-based approach in which visual information is used to retrieve the observable deformation on the tissue surface after applying force. This information, together with time, is the input to the second module, V-ANFIS, which attempts to handle visual uncertainty and obtain the final tissue surface displacements. Finally, the third module is focused on estimating the

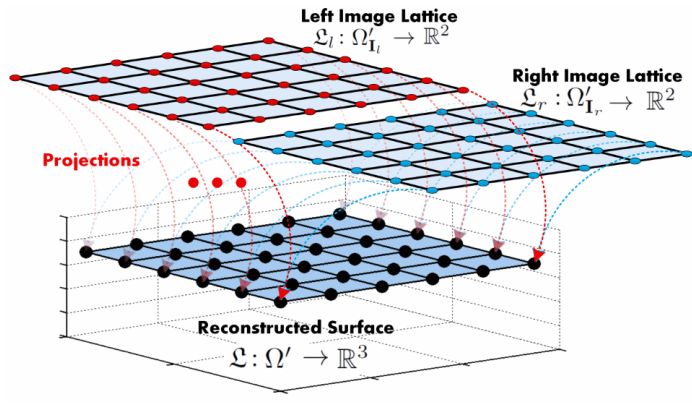

Figure 2: Tissue's surface reconstruction is achieved from the projection of the corresponding points of the lattices defined on each stereo-pair image.

applied force, analyzing the relation between visual information and kinematic variables and using the capability of a Recurrent Neural Network (RNN) on doing temporal processing.

This paper is focused on the development of the V-ANFIS approach. Since the proposed approach attempts to handle visual uncertainty on the reconstructed deformation structure, the next subsection is devoted to explain how the parametrization of the tissue surface is performed. Specific functioning of the third module can be found in [31].

\subsection{Vision-Based Approach: Tissue Surface Parametrization}

Observable deformations on the tissue surface are calculated using the visual information given by a stereo camera. Consider a stereo-pair image acquired at time $t$, where left and right images are defined by $\mathbf{I}_{l}: \Omega_{\mathbf{I}_{l}} \rightarrow \mathbb{R}^{2}$ and $\mathbf{I}_{r}: \Omega_{\mathbf{I}_{r}} \rightarrow \mathbb{R}^{2}$, and where $\Omega_{(\cdot)}$ is the bounded image domain. Since the surgeon is only interested in the zone to be repaired, a region of interest is defined on the tissue surface via a set of control points, $\mathbf{P}$, which belongs to a uniform lattice. Let $\mathfrak{L}_{l} \in \Omega_{\mathbf{I}_{l}}^{\prime}$ and $\mathfrak{L}_{r} \in \Omega_{\mathbf{I}_{r}}^{\prime}$ be the lattices defined on $\mathbf{I}_{l}$ and $\mathbf{I}_{r}$, respectively, where $\Omega_{(\cdot)}^{\prime}$ is a subdomain of $\Omega_{(\cdot)}$. The three-dimensional lattice, $\mathfrak{L} \in \mathbb{R}^{3}$, is obtained from the projections of the corresponding points of $\mathfrak{L}_{l} \in \mathbb{R}^{2}$ and $\mathfrak{L}_{r} \in \mathbb{R}^{2}$ (see Figure 2).

After that, the process for obtaining the changes produced on the tissue surface, when a force is applied, is defined as an energy minimization problem in which the $l 2-$ regularized optimization class is applied. The reason why this class is used is twofold: the optimization process is straightforward, i.e. the energy functional is differentiable and derivatives are continuous, and it demands low computational cost. Assuming that the input images have been rectified, the deformed structure is reconstructed by finding the set of control points, $\mathbf{P}$, in which an optimal transformation is obtained by minimizing the 


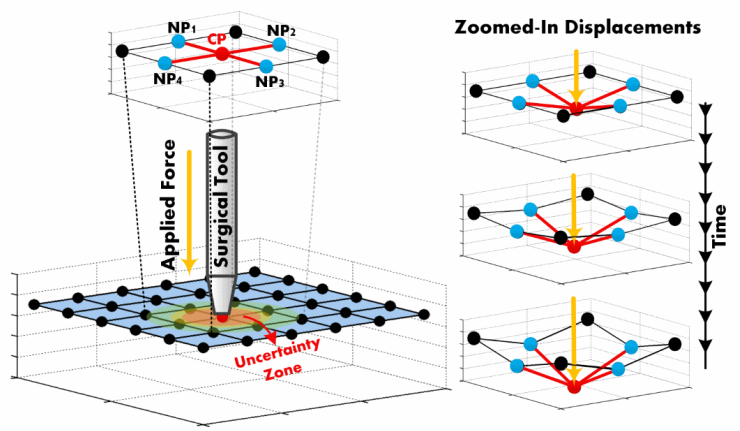

Figure 3: Surface deformation as a result of the force applied by surgical tool (left side). Uncertainty zone zoomed-in displacements over-time are also shown (right side).

following expression:

$$
\mathbf{E}_{d c r}\left(\mathbf{I}_{l}(\mathbf{d}(\mathbf{x} ; \mathbf{P})+\mathbf{x}), \mathbf{I}_{r}(\mathbf{x})\right)+\gamma \mathbf{E}_{p n l}(\mathbf{d}(\mathbf{x} ; \mathbf{P}))
$$

where $\mathbf{E}_{d c r}$ is a discrepancy term that allows measuring the level of alignment between $\mathbf{I}_{l}$ and $\mathbf{I}_{r}$; $\mathbf{E}_{p n l}$ is the penalization term that allows obtaining a plausible transformation fulfilling Hadamard's postulate [32]; $\gamma$ gives a balance between both terms; and $\mathbf{x}$ is a vector. In order to carry out the mapping, $\mathbf{d}_{m}$, at any position $\mathbf{x}$, the tensor product of basis splines, $\xi_{(\cdot)}$, of degree $n$ at $d$-dimensional space are used as shown in Eq. 2:

$$
\mathbf{d}_{m}(\mathbf{x} ; \mathbf{P})=\sum_{j_{1}=0}^{n} \ldots \sum_{j_{d}=0}^{n} \overbrace{\mathbf{P}_{j_{1}, \ldots, j_{d}}}^{\prod_{\prod_{\text {tensor product }} \xi_{k}\left(\mathbf{x}_{k}\right)}^{d o n t o l} \text { points }}
$$

Detailed explanation of the parametrization of the tissue's surface can be found in our previous works [33], [34].

\section{Handling Visual Uncertainty}

Uncertainty abounds in every phase of computer vision. Therefore, for a computer vision system to be robust, it must have at its disposal the machinery allowing vagueness representation. Moreover, from a theoretical point of view, the techniques used during the system development must anticipate vagueness effects in order to obtain a correct interpretation of the results [35]. In the problem at hand, while displacements on the tissue surface can be accurately retrieved using visual information, a challenging problem appears when part of the target is occluded by the surgical instrument. These occlusions entail lack of knowledge and information about a portion of tissue which must be retrieved (see Figure 3).

In this work, the problem of recovering the lost observable deformation in a period of time, when the camera is blocked, is addressed by using the so called V-ANFIS approach. V-ANFIS is the

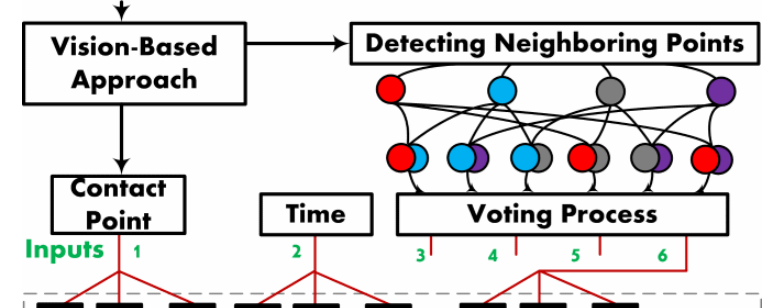

$S_{1} S_{2}^{1} \ldots S_{N_{2}}^{2} S_{1}^{2} S_{2}^{2} \ldots S_{N_{2}}^{2} \ldots S_{1}^{6} S_{2}^{6} \cdots S_{N_{2}}^{6}$

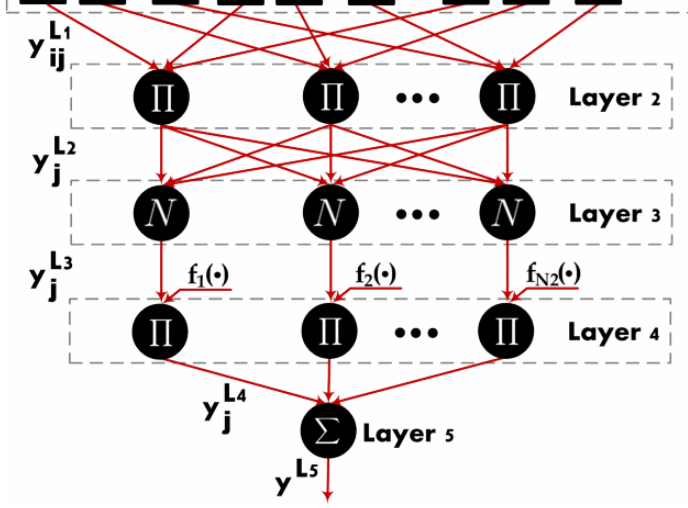

Figure 4: Architecture of the proposed V-ANFIS approach to handle visual uncertainty.

combination of a voting process and the ANFIS soft computing hybrid strategy. For a better understanding of the proposed V-ANFIS algorithm, first a set of conventions and assumptions are presented.

CP: contact point between the tissue surface and surgical tool. This is the point having the largest displacement, in Z-direction, within the three-dimensional lattice $\mathfrak{L} \in \mathbb{R}^{3}$.

$\mathbf{F}^{(t)} \in \mathbb{R}^{-} \cup\{0\}$ : force applied on $\mathbf{C P}$ and propagated through $\mathfrak{L}$.

GP: geometric information provided by a robot manipulator about the end effector point.

$\mathbf{N P}_{i}: i-t h$ neighboring point that has direct connection with $\mathbf{C P}$ and belongs to $\mathfrak{L}$.

C: combination matrix used to carry out a pair-search which identifies points that need correction.

Observation 1 Note that forces are applied in $Z-$ direction, which allows making the assumption that neighboring points should have equal displacements.

The equal neighboring points' displacements assumption in observation 1 is useful to find points that have error over time. A discordance between the points will tell us which points should be corrected. In other words, we assign displacement value, to all points, based on the concordance of the majority. 


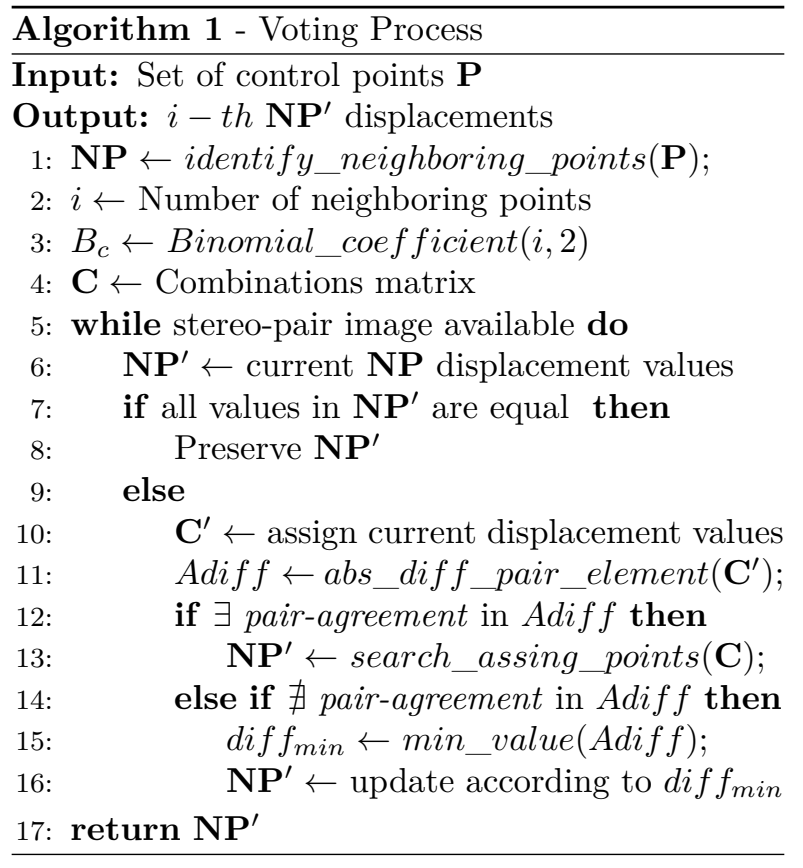

\subsection{V-ANFIS Strategy}

The visual uncertainty problem is tackled in this paper using a strategy that we call V-ANFIS. This is divided into two main steps. The former is a voting process that allows decreasing the neighboring points error using combinatorial and agreement processes. The latter is a prediction step based on the ANFIS properties, in which the main idea is to estimate the lost information, when an occlusion occurs at contact point, using patterns of the available data.

In order to deal with the propagated uncertainty around the contact point, $\mathbf{C P}$, the neighboring points go through a voting process. This process is explained in Algorithm 1 and the following definitions are given for clarification.

Definition 1 Let $i$ be a positive integer denoting the number of neighboring points, $B_{c}=\left(\begin{array}{l}i \\ b\end{array}\right)$ the binomial coefficient, and $\mathbf{C}$ the combinations matrix, having $b$ columns, and $B_{c}$ rows. Then, a pair-element is a combination in $\mathbf{C}$.

Definition 2 (Pair-agreement). Given a pair-element in $\mathbf{C}$, we say that it fulfills the pair-agreement condition if and only if at current time both points have the same value.

Once uncertainty has been taken into account, the prediction of the applied forces is carried out using an Adaptive Neuro-Fuzzy Inference System (ANFIS). ANFIS [24] is an approach that combines the benefits of both Fuzzy Systems (semantic transparency) and Neural Networks (adaptability). In addition, ANFIS offers a better learning step that converges faster than using just NN, does not require a bigger training set, and provides better interpretation of the internal process. It also has an equivalent functionality to a Fuzzy Inference

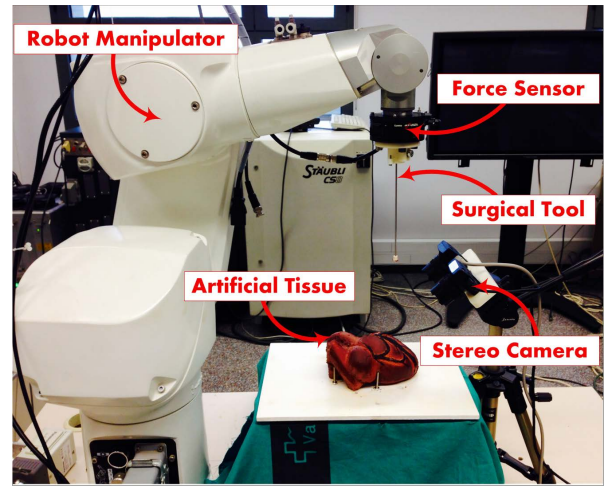

Figure 5: Experimental platform used to validate the proposed approach.

System (FIS) in which a set of if-then rules are used [24]. Specifically, in this work we use the capability of ANFIS as universal approximator [38], in which mathematical proof involves looking the Stone-Weierstrass theorem [39].

The ANFIS architecture used in this work is illustrated in Figure 4, in which $N$ inputs are given. In Layer $1\left(\mathbf{L}_{1}\right)$, the fuzzification layer, the membership grade of the input variables is specified using a gaussian function, $\mu(\cdot)$, expressed as:

$$
\mu_{\mathbf{S}_{j}^{i}}\left(x_{i}\right)=\exp \left(\frac{-1}{2}\left(\frac{x_{i}-c_{j}^{i}}{\sigma_{j}^{i}}\right)^{2}\right)
$$

where $c$ denotes the center and $\sigma$ the adjustable width of the Gaussian. Here, $i$ and $j$ stand for the $i$-neuron and $j$-rule, while $\mathbf{S}_{j}^{i}$ are the regions of the input space partitioning. In this work, we use scatter partition since it is well-known by its effective performance in comparison with other approaches [37]. It also avoids both the dimensionality problem and data interpretability degradation. The output of this layer, $\mathbf{y}^{\mathbf{L}_{1}}$, is written as follows:

$$
\mathbf{y}_{i j}^{\mathbf{L}_{1}}=\mu_{\mathbf{s}_{j}}^{i}\left(x_{i}\right) \text { for } i=1, \ldots, N ; j=1, \ldots, N_{2}
$$

where $N$ refers to the number of input variables and $N_{2}$ the number of rules. Layer $2\left(\mathbf{L}_{2}\right)$ is a set of neurons in which the product operator, $A N D$, is used to obtain the belief strength, i.e. the firing strength, of the rules. The output, $\mathbf{y}^{\mathbf{L}_{2}}$, is calculated as:

$$
\mathbf{y}_{j}^{\mathbf{L}_{2}}=\prod_{i=1}^{N_{2}} \mathbf{y}_{i j}^{\mathbf{L}_{1}}
$$

In Layer 3, the normalization of the firing strengths are obtained as:

$$
\mathbf{y}_{j}^{\mathbf{L}_{3}}=\frac{\mathbf{y}_{j}^{\mathbf{L}_{2}}}{\sum_{n_{2}=1}^{N_{2}} \mathbf{y}_{n_{2}}^{\mathbf{L}_{2}}} \text { for } j=1, \ldots, N_{2}
$$

The fourth layer executes the consequent part of the fuzzy rules. Its output, $\mathbf{y}_{j}^{\mathbf{L}_{4}}$, is a function of 

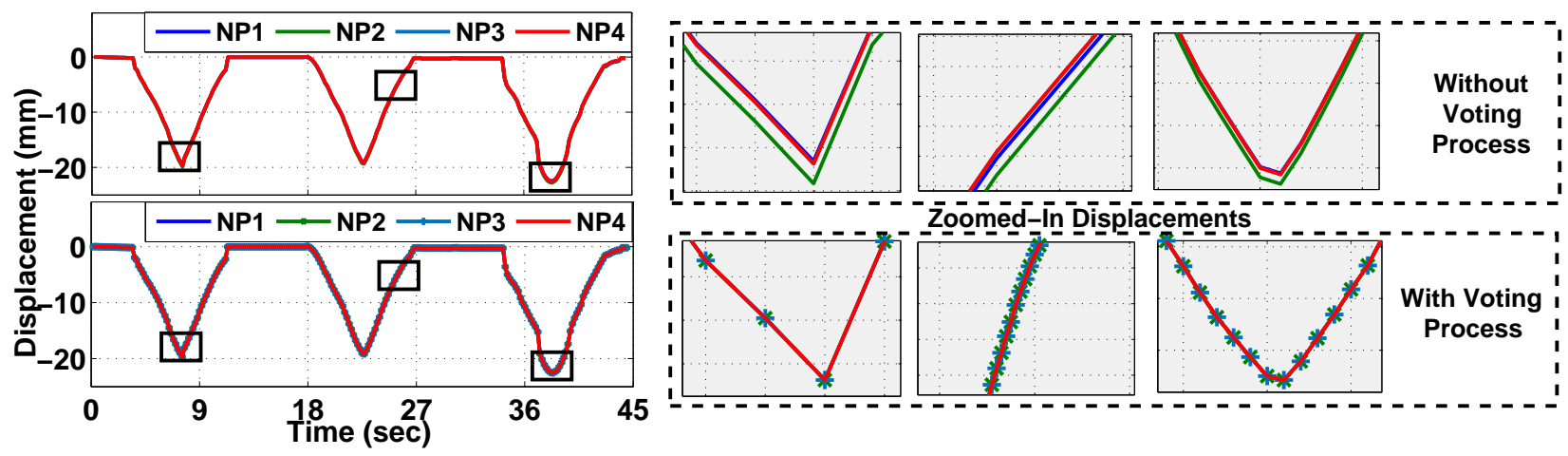

Figure 6: Retrieved displacements, in z-direction, of the four immediate neighboring points are plotted first without voting process correction (top) then with voting process (bottom).
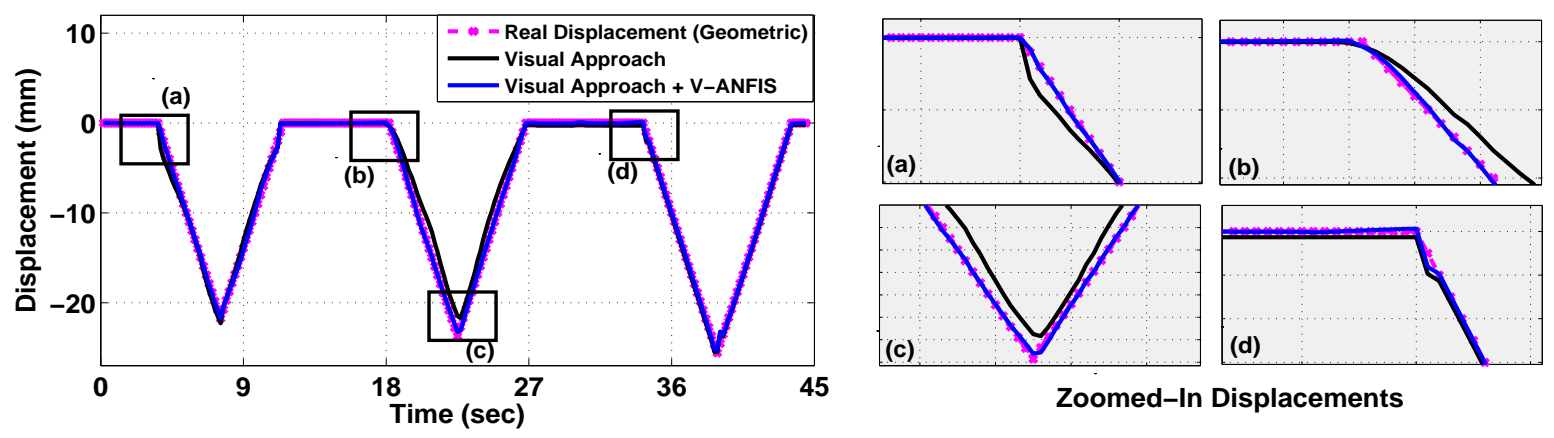

Figure 7: (From left to right) Comparison of displacements, at contact point, between real-geometric measure and visual approach with and without V-ANFIS. Zoomed-In displacements are also shown in order to observe the significant improvement when V-ANFIS is applied.

the consequent parameters, $\mathfrak{f}(\cdot)$, and the previous output layer expressed as:

$$
\mathbf{y}_{j}^{\mathbf{L}_{4}}=\mathbf{y}_{j}^{\mathbf{L}_{3}} \mathfrak{f}_{j}\left(x_{i}\right)
$$

Finally, ANFIS's output, $\mathbf{y}^{\mathbf{L}_{5}}$, is a single node which sums up the outputs of the previous layer.

$$
\mathbf{y}^{\mathbf{L}_{5}}=\sum_{j=1}^{N_{2}} \mathbf{y}_{j}^{\mathbf{L}_{4}}
$$

Working under the assumption that geometric information, GP, provides accurate values, it is used as the ground truth during the training phase.

\section{Experimental Results}

A realistic scenario has been used to analyze our approach, this, together with simulated tests, validate the viability and efficacy of our proposed approach. A pair of stereo-cameras (IDS) and a robot manipulator (Stäubli RX60B) have been employed as part of our scenario. Moreover, an artificial tissue, with the same mechanical properties of soft human tissues, is used. To compare our proposed approach with a realistic force measure, an ATI Gamma force sensor is added to the robotic manipulator (see Figure 5).
Accurate estimation of the observed deformable tissue displacement is very important in order to improve the applied force estimation. Thus, we proposed the use of a voting process to reduce the error that occurs at the neighboring points. Figure 6 shows the displacement values at neighboring points with and without applying the voting process. Zoomed in views show the significant improvement after applying the voting step, which brings the displacements of all neighboring points into a complete agreement.

To evaluate the overall accuracy of our approach, we recorded three different readings of the displacement value at contact point. The first reading corresponds to the real displacement, i.e. Geometric information, obtained from the robot manipulator. The second and third readings were estimated by applying the visual approach with and without the V-ANFIS. Figure 7, left side, shows a plot comparing the recorded displacement values. We can see that applying V-ANFIS brings the estimated values closer to the real-geometric measure. This assertion can be better appreciated in the four zoomed-in plots at right side of Figure 7. Moreover, the absolute error between the real measure and the readings is illustrated in Figure 8. Needless to say that V-ANFIS outperformed the other two alternatives and successfully reduced the 

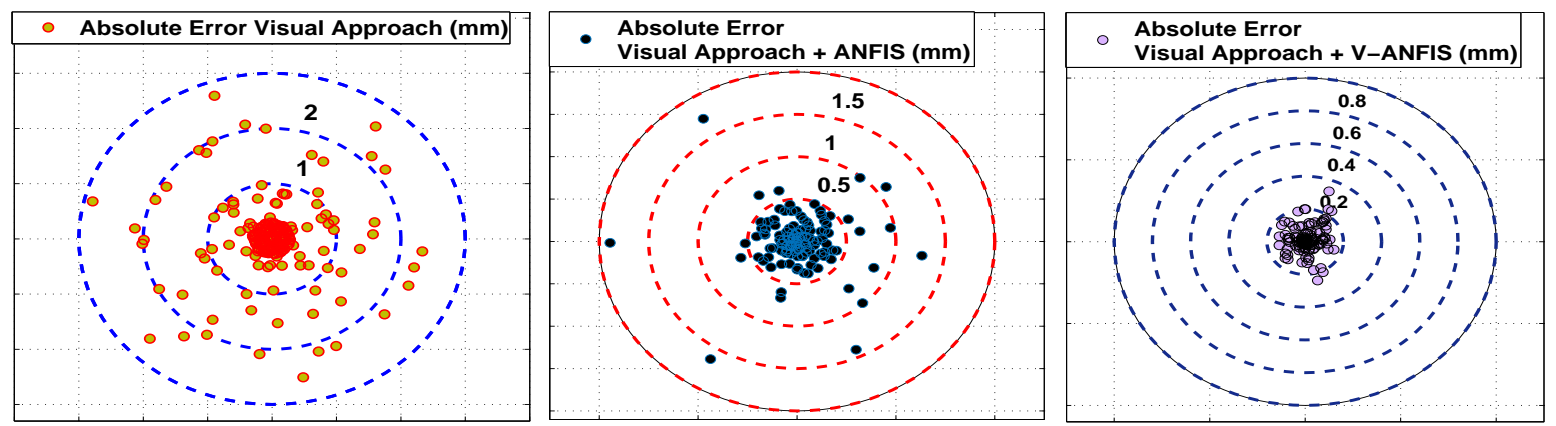

Figure 8: Absolute error of estimated contact point displacements given by visual approach alone (left plot), visual approach with ANFIS (middle plot), and visual approach with V-ANFIS (right plot).
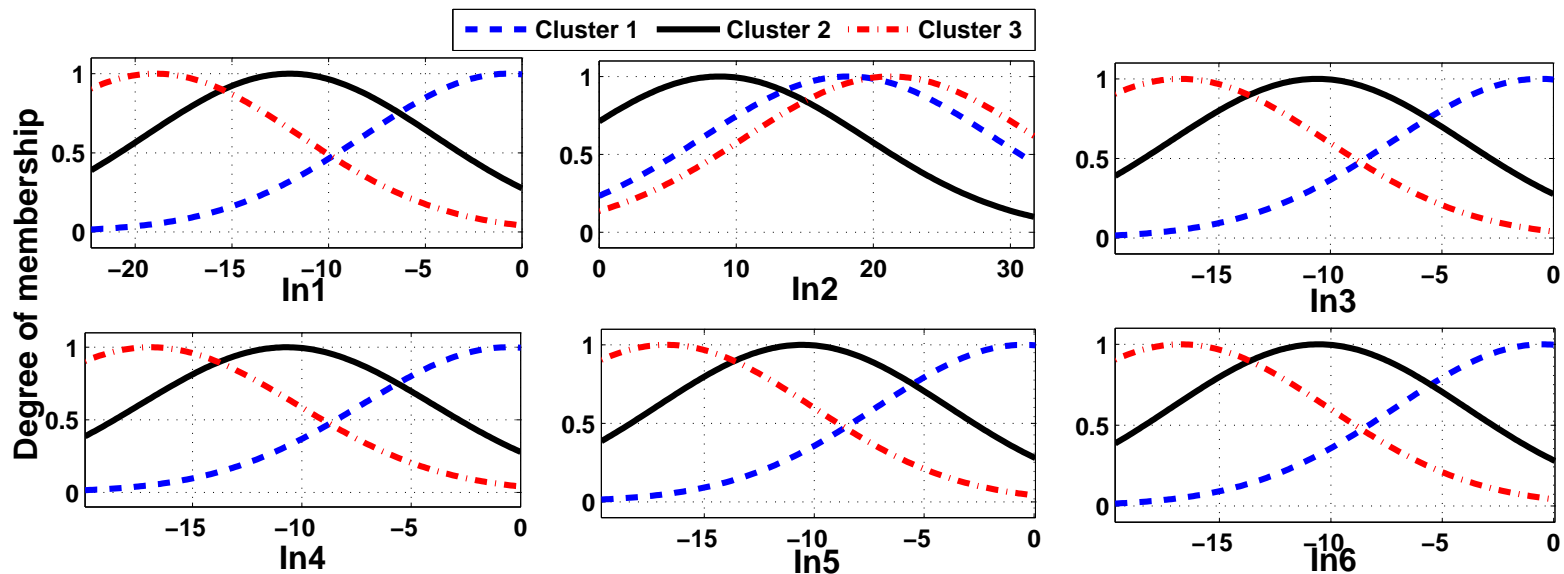

Figure 9: Learned membership functions used to cover the input space. It can be seen that three clusters were necessary for each input, In.

absolute error of the samples from $2 \mathrm{~mm}$ to less than $0.2 \mathrm{~mm}$.

Since the goal is to find those membership functions (MFs) that best fit our data, the learned MFs after training are shown in Figure 9, initial ones were defined by a scatter partition. Finally, the real force measures, given by the sensor, and the estimated measures, given by V-ANFIS+RNN, are shown in Figure 10. As can be appreciated, the reported force measures provide an excellent approximation with respect to the real ones, which proves the effectiveness of the proposed approach. It is worth mentioning that we displayed a 45 seconds time window as it clearly illustrates the algorithm's performance and shows that while $t \rightarrow \infty$, the error does not increase and remains stable. Nonetheless, the stability of our approach was validated in longer recordings from $1140 \mathrm{sec}$. to $1560 \mathrm{sec}$.

Besides improving the displacement estimation and having in mind that our ultimate aim is to improve the estimated force and bring it closer to the target values, we have checked the effect of $\mathrm{V}$-ANFIS in the applied force estimation. Table 1 shows the root mean square error (RMS), in regards to force estimation, of our approach along with errors reported by previous studies. The RMS error of our approach is 0.0315 , which shows
Table 1: Performance analysis of existing and proposed approaches.

\begin{tabular}{|l|c|c|}
\hline Method & RMS Error(N) & \% Imp. \\
\hline \hline Yip et al. [9] & 0.13 & 75.77 \\
Aviles et al. [21] & 0.0627 & 49.76 \\
Faragasso et al. [11] & 0.1355 & 76.75 \\
Noohi et al. [18] & 0.07 & 55 \\
Our Approach & $\mathbf{0 . 0 3 1 5}$ & - \\
\hline
\end{tabular}

that it outperforms all the other approaches under comparisons. Moreover, it is worth mentioning that our approach offers a significant percentage of improvement, ranging from about $50 \%$ to $77 \%$. These observations prove that handling visual uncertainty has a positive impact and ultimately improves the applied force estimation.

\section{Conclusions}

During robotic assisted surgeries, both robustness and accuracy are of great importance. Therefore, even slight uncertainty in the retrieved visual information may result in life hazards. Based on such considerations, in this study, a novel design of a soft computing based prediction model 


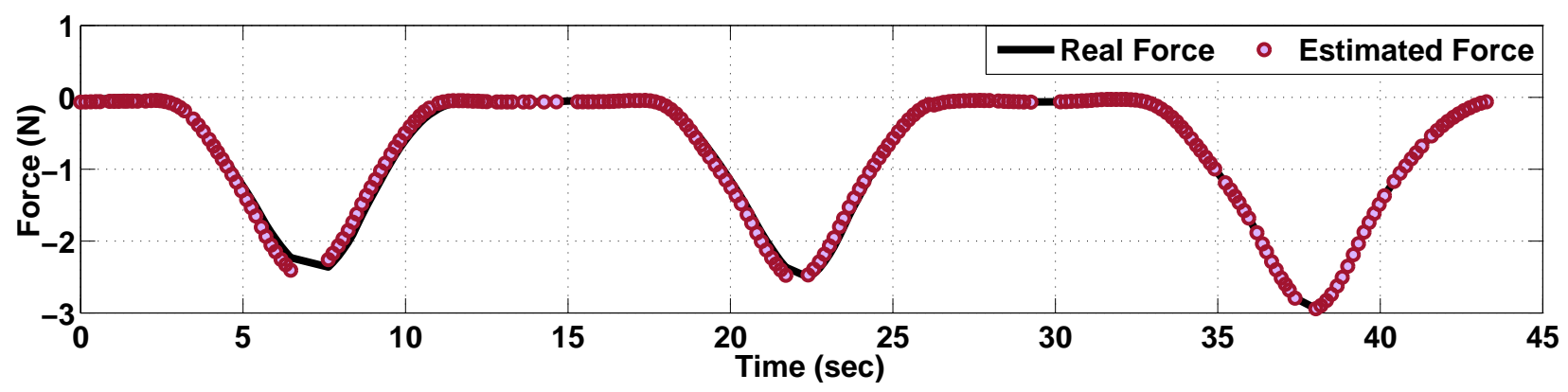

Figure 10: Comparison of the real and estimated force values, taking into account visual uncertainty.

was developed. The proposed model, V-ANFIS, combines the predictive capabilities of adaptive neuro-fuzzy inference systems with the corrective benefits of agreements and combinatorial processes. The superior performance of the proposed approach, compared to existing techniques, is demonstrated through experiments conducted on a realistic environment. Observations showed a reduction on absolute error of retrieved surface displacement from $2 \mathrm{~mm}$ to as little as $0.2 \mathrm{~mm}$.

Furthermore, when compared to existing approaches reported for force estimation, the addition of V-ANFIS to the proposed system provided a favorable percentage of improvement ranging from about $50 \%$ to $77 \%$. These results confirm that soft computing can effectively handle visual uncertainty and offers a cost-effective solution.

\section{Acknowledgment}

This work was supported by a FPU national scholarship from the Spanish Ministry of Education with reference AP2012-1943. Also, it is part of the project DPI2011-29660-Co4-01 and DPI2011-29660-CO4-03 MINECO and with FEDER funds EC.

\section{References}

[1] E.B. Wilson, H. Bagshahi, and V.D. Woodruff, Overview of General Advantages, Limitations, and Strategies Book Chapter, Robotics in General Surgery, Springer, 2014.

[2] A.M. Okamura, Haptic feedback in robot-assisted minimally invasive surgery, Current Opinion in Urology, 19(1):102-10\%, 2009

[3] B. Bethea, A. Okamura, M. Kitagawa, T. Fitton, S. Cattaneo, V. Gott, W. Baumgartner, D. Yuh, Application to haptic feedback to robotic surgery, J Laparoendosc Adv Surg Tech A. 191-195, 2004.

[4] S. De, J. Rosen, A. Dagan, B. Hannaford, P. Swanson, M. Sinanan, Assessment of tissue damage due to mechanical stresses, The Int. Journal of Robotics Research, 2007.
[5] N. Famaey, E. Verbeken, S. Vinckier, B. Willaert, P. Herijgers, P. and J. Vander Sloten, In vivo soft tissue damage assessment for applications in surgery, Medical Engineering and Physics, 2009.

[6] G. Tholey, J. Desai and A. Castellanos, Force feedback plays a significant role in minimally invasive surgery, Annals of surgery, 2005.

[7] C. Pacchierotti, A. Tirmizi and D. Prattichizzo, Improving transparency in teleoperation by means of cutaneous tactile force feedback, $A C M$ Transactions on Applied Perception, 2014.

[8] K. Zareinia, Y. Maddahi, C. Ng, N. Sepehri and G.R. Sutherland, Performance evaluation of haptic hand-controllers in a robot-assisted surgical system, Int J Med Robotics Comput Assist Surg, 2015.

[9] M.C. Yip, S.G. Yuen and R.D. Howe, A Robust Uniaxial Force Sensor for Minimally Invasive Surgery, IEEE Trans. on Biomedical Engineering, pp. 1008-1011, 2010.

[10] P. Puangmali, H. Liu, L.D. Seneviratne, P. Dasgupta and K. Althoefer, Miniature 3-Axis Distal Force Sensor for Minimally Invasive Surgical Palpation, IEEE Trans. on Mechatronics, pp. 646-656, 2012.

[11] A. Faragasso, J. Bimbo, Y. Noh, A. Jiang, S. Sareh, H. Liu, H.A. Wurdemann and K. Althoefer, Novel Uniaxial Force Sensor based on Visual Information for Minimally Invasive Surgery, IEEE International Conference on Robotics and Automation, 2014.

[12] K.L. Helton, B.D. Ratner and N.A. Wisniewski, Biomechanics of the Sensor-Tissue Interface-Effects of Motion, Pressure, and Design on Sensor Performance and the Foreign Body Response-Part I: Theoretical Framework, Journal of Diabetes Science, Vol. 5, 2011.

[13] S. Sokhanvar, J. Dargahi, S. Najarian and S. Arbatani, Clinical and Regulatory Challenges for Medical Devices Tactile Sensing and Displays, Haptic Feedback for Minimally Invasive Surgery and Robotics, Wiley Publications, 2012.

[14] M.A. Greminger and B.J. Nelson, Vision-based force measurement IEEE Trans on Pattern Analysis and Machine Intelligence, 2004. 
[15] J. Kim, F. Janabi-Sharifi and J. Kim, A Haptic Interaction Method Using Visual Information and Physically Based Modeling IEEE Transactions on Mechatronics, Vol. 15, No. 4, 2010.

[16] J. Jung, J. Lee and K. Huh, Robust contact force estimation for robot manipulators in three-dimensional space Journal of Mechanical Engineering Science, vol. 220, 2006.

[17] M. Ammi, H. Ladjal and A. Ferreira, Evaluation of 3D Pseudo-Haptic Rendering using Vision for Cell Micromanipulation, IEEE/RSJ International Conference on Intelligent Robots and Systems, 2006.

[18] E. Noohi, S. Parastegari and M. Zefran, Using Monocular Images to Estimate Interaction Forces During Minimally Invasive Surgery, IEEE International Conference on Intelligent Robots and Systems, 2014.

[19] A. E. Kerdok, S. M. Cotin, M. P. Ottensmeyer, A. M. Galea, R. D. Howe, and S. L. Dawson, Truth cube: Establishing physical standards for soft tissue simulation, Medical Image Analysis, 2003.

[20] F. Karimirad, S. Chauhan and B. Shirinzadeh, Vision-based force measurement using neural networks for biological cell microinjection Journal of Biomechanics, Volume 47, Issue 5, pp. 1157-1163, 2014.

[21] A.I. Aviles, A. Marban, P. Sobrevilla, J. Fernandez and A. Casals, A Recurrent Neural Network Approach for 3D Vision-Based Force Estimation, IEEE International Conference on Image Processing Theory, Tools and Applications, 2014.

[22] A. Torres and J.J Nieto, Fuzzy Logic in Medicine and Bioinformatics, Journal of Biomedicine and Biotechnology, 2006.

[23] S.T. Karaginnis, A.I Dounis, T. Chalastras, P. Tiropanis and D. Papachristos, Design of Expert System For Search Allergy and Selection of the Skin Test Using CLIPS, Int. J. of Information Technology, 2007.

[24] J.-S.R. Jang, ANFIS: adaptative-network-based fuzzy inference system IEEE Transactions on Systems, Man and Cybernetics, vol. 23 no. 3, 1993.

[25] C. Kalaiselvi and G. M. Nasira. A New Approach for Diagnosis of Diabetes and Prediction of Cancer Using ANFIS. Computing and Communication Technologies World Congress on. IEEE, 2014.

[26] A. Kolus, P.A. Dube, D. Imbeau, R. Labib, and D. Dubeau, Estimating oxygen consumption from heart rate using adaptive neuro-fuzzy inference system and analytical approaches, Applied ergonomics, 2014.

[27] A. Thiyagarajan, P.R. Rajasekaran and K. Subramanian, ANFIS-EM approach for PET brain image reconstruction, Int. J. Imaging
Syst. Technol., 2015.

[28] T. Nguyen, A. Khosravi, D. Creighton and S. Nahavandi, Medical data classification using interval type-2 fuzzy logic systemand wavelets, Journal in Applied Soft Computing, 2015.

[29] A. Mozaffari and S. Behzadipour, A modular extreme learning machine with linguistic interpreter and accelerated chaotic distributor for evaluating the safety of robot maneuvers in laparoscopic surgery. Elsevier Journal in Neurocomputing, 2015.

[30] A. Mozaffari, S. Behzadipour and M. Mehdi Kohani, Identifying the tool-tissue force in robotic laparoscopic surgery using neuro-evolutionary fuzzy systems and a synchronous self-learning hyper level supervisor. Elsevier Journal in Applied Soft Computing, 2014.

[31] A.I. Aviles, S. Alsaleh, P. Sobrevilla and A. Casals., Sensorless Force Estimation using a Neuro-Vision-Based Approach for Robotic-Assisted Surgery. To appear in International IEEE EMBS Conference on Neural Engineering., 2015.

[32] J. Hadamard, Lectures on the Cauchy Problems in Linear Partial Differential Equations, New Haven Yale University Press, 1923.

[33] A.I. Aviles, P. Sobrevilla and A. Casals, An Approach for Physiological Motion Compensation in Robotic-Assisted Cardiac Surgery, Journal in Experimental and Clinical Cardiology, 2014.

[34] A.I. Aviles, P. Sobrevilla and A. Casals, Unconstrained $\ell 1-$ Regularized Minimization with Interpolated Transformation for Heart Motion Compensation, International Conference of the IEEE Engineering in Medicine and Biology Society, 2014.

[35] P. Sobrevilla and E. Montseny, Fuzzy sets in computer vision: An overview Mathware and Soft Computing pp. 71-83, 2003.

[36] A.N. Tikhonov, On solving incorrectly posed problems and method of regularization, Dokl. Acad. Naukl USSR, 151(3), pp. 501-504, 1963.

[37] S.Y. Ho, H.M. Chen, S.J. Ho and T.K. Chen, Design of accurate classifiers with a compact fuzzy-rule base using an evolutionary scatter partition of feature space IEEE Transactions on Systems, Man and Cybernetics, vol. 34 no. 2, 2004.

[38] J.-S.R. Jang, C.-T. Sun and E. Mizutani, Neuro-Fuzzy and Soft Computing: A Computational Approach to Learning and Machine Intelligence Prentice Hall, Inc., 1997.

[39] M.H. Stone, The Generalized Weierstrass Approximation Theorem Mathematics Magazine 21(4), 1948. 